\title{
Gut microbiota contributes to the development of endometrial glands in gilts during the ovary-dependent period
}

Baoyang Xu $u^{1,2,3}$, Wenxia Qin ${ }^{1,2,3}$, Yiqin Yan ${ }^{1,2,3}$, Yimei Tang ${ }^{1,2,3}$, Shuyi Zhou ${ }^{1,2,3}$, Juncheng Huang ${ }^{1,2,3}$, Chunlin Xie ${ }^{1,2,3}$, Libao $\mathrm{Ma}^{1,2,3^{*}}$ and Xianghua Yan ${ }^{1,2,3^{*}}$

\begin{abstract}
Background: The hyper-prolificacy Meishan gilts achieved a superior endometrial gland development (EGD) than white crossbred gilts during the ovary-independent period (before $60 \mathrm{~d}$ of age). Then, the EGD continues under the management of ovary-derived steroid hormones that regulated by gut microbiota (after $60 \mathrm{~d}$ of age). However, whether Meishan gilts' superiority in EGD lasting to the ovary-dependent period (after $60 \mathrm{~d}$ of age) and the role of gut microbiota in this period both remain unclear.

Methods: Meishan gilts and Landrace $x$ Yorkshire $(L x Y)$ gilts were raised under the same housing and feeding conditions until sexual maturity and then we compared their EGD and gut microbiota. Meanwhile, we transplanted fecal microbiota from Meishan gilts to $L \times Y$ gilts to explore the role of gut microbiota in EGD. We sampled plasma every 3 weeks and collected the uterus, ovary, liver, and rectal feces after the sacrifice. We then determined the hormone concentrations and expressions of the EGD-related genes. We also profiled the gut microbiota using 165 rDNA sequencing and metabolites of plasma and liver tissue using untargeted metabolomics. Finally, the correlation analysis and significant test was conducted between FMT-shifted gut microbes and EGD-related indices.

Results: Meishan gilts have larger endometrial gland area $(P<0.001)$, longer uterine horn length $(P<0.01)$ but lighter uterine horn weight $(P<0.05)$, a distinctive gut microbiota compared with $L \times Y$ gilts. Fecal microbiota transplantation (FMT) increased endometrial gland area $(P<0.01)$. FMT markedly shifted the metabolite profiles of both liver and plasma, and these differential metabolites enriched in steroid hormone biosynthesis pathway. FMT increased estradiol and insulin-like growth factor 1 but decreased progesterone dynamically. FMT also increased the expression of the EGD-related genes estrogen receptor 1 gene, epithelial cadherin, and forkhead box protein A2. There is a significant correlation between FMT-shifted gut microbes and EGD-related indices.
\end{abstract}

Conclusion: Sexually matured Meishan gilts achieved a superior EGD than LXY gilts. Meanwhile, gut microbiota contribute to the EGD potentially via regulating of steroid hormones during the ovary-dependent period.

Keywords: Endometrial gland, Fecal microbiota transplantation, Gut microbiota, Meishan gilts, Steroid hormones, Untargeted metabolomics, Uterus

\footnotetext{
*Correspondence: malibao@mail.hzau.edu.cn; xhyan@mail.hzau.edu.cn

'State Key Laboratory of Agricultural Microbiology, College of Animal

Sciences and Technology, Huazhong Agricultural University, Wuhan 430070,

Hubei, China

Full list of author information is available at the end of the article
}

(c) The Author(s). 2021 Open Access This article is licensed under a Creative Commons Attribution 4.0 International License, which permits use, sharing, adaptation, distribution and reproduction in any medium or format, as long as you give appropriate credit to the original author(s) and the source, provide a link to the Creative Commons licence, and indicate if changes were made. The images or other third party material in this article are included in the article's Creative Commons licence, unless indicated otherwise in a credit line to the material. If material is not included in the article's Creative Commons licence and your intended use is not permitted by statutory regulation or exceeds the permitted use, you will need to obtain permission directly from the copyright holder. To view a copy of this licence, visit http://creativecommons.org/licenses/by/4.0/. The Creative Commons Public Domain Dedication waiver (http://creativecommons.org/publicdomain/zero/1.0/) applies to the data made available in this article, unless otherwise stated in a credit line to the data. 


\section{Background}

Mammalian uteri contain glands in the endometrium that synthesize or transport and secrete substances essential for survival and development of the conceptus (embryo/fetus and associated extraembryonic membranes), thus contributing to female prolificacy [1, 2]. Endometrial adenogenesis is primarily a postnatal event in sheep, pigs, and rodents [3]. At birth, no endometrial glands are present in uterus and then within $7 \mathrm{~d}$, endometrial glands begin to differentiate, and they continue to differentiate during the next several months [3]. Neonatal porcine uterine development occurs in an ovaryindependent manner before, and in an ovary-dependent manner after, day 60 of neonatal life $[4,5]$. In ovarydependent development period, ovarian hormones estradiol and progesterone play crucial roles in endometrial glands development [5]. Estradiol is a primary mitogen for uterine epithelium and its increase around estrous induces proliferation of both uterine luminal epithelium and glandular epithelium [5]. Neonatal estradiol exposure alters uterine morphology and endometrial transcriptional activity in prepubertal gilts [6]. Progesterone can inhibit estradiol actions in stimulation of uterine epithelial proliferation through epithelial progesterone receptors [5, 7]. This relationship between estradiol and progesterone may regulate the endometrial gland development and uterine function.

Meishan pigs as a Chinese indigenous breed is characterized by its prolificacy, producing an average of three to four more piglets per litter than European breeds [8]. However, the underlying mechanism by which Meishan pigs achieve superior litter size remains not fully understood. Christenson et al. found that Meishan gilts' uterus secreted more abundance of endometrial proteins, which increased in association with endometrial gland development before day 60 of neonatal life [9]. Further histological analysis validated that Meishan gilts achieved a superior endometrial gland development than white crossbred gilts at $60 \mathrm{~d}$ of age [9]. However, the further comparative morphological study of their endometrial gland development after $60 \mathrm{~d}$ of age remains undetermined. A recent study showed that the Meishan sows of high-prolificacy both have increased fecal microbiota diversity and fecal steroid hormones estradiol and progesterone levels than low-prolificacy ones, which may contribute to the improvement of sows' reproductive performance [10]. Interestingly, microbiota that harbor inside the host intestine function as a vital endocrine organ get involved in regulating steroid hormones estradiol and progesterone $[11,12]$.

Given that the vital role of steroid hormones estradiol and progesterone in endometrial glands development during the ovary-dependent period [7, 13, 14], we hypothesis that gut microbiota get involved in the superior endometrial gland's development of prolific Meishan gilts than white crossbred gilts during the ovarydependent period. Fecal microbiota transplantation (FMT) involves the transfer of donor fecal microbiome to a recipient in order to establish a more desirable or donor-like gut microbiome $[15,16]$. FMT provides an effective approach to link gut microbiota and host phenotype $[15,17]$. To test our hypothesis, we compared the uterine development and gut microbiota between sexually matured Meishan gilts and crossbred Landrace $\times$ Yorkshire $(\mathrm{L} \times \mathrm{Y})$ gilts under the same housing and feeding conditions. Meanwhile, for exploring the role of gut microbiota in endometrial glands development, we transplanted fecal microbiota of Meishan gilts to recipient $\mathrm{L} \times \mathrm{Y}$ gilts.

\section{Methods}

\section{Experimental design and animals}

All experiments involving swine were carried out under the recommendations of the Guide for the Care and Use of Laboratory Animals Monitoring Committee of Hubei Province, China, and the protocol was approved by the Scientific Ethics Committee of Huazhong Agricultural University (approval numbers HZAUSW-2018-015). All efforts were made to minimize animal suffering. Eight Meishan gilts of age $95 \pm 7 \mathrm{~d}$ from the National Conservation Farm of Meishan Pigs (Jiading, Shanghai) and 14 Landrace $\times$ Yorkshire $(\mathrm{L} \times \mathrm{Y})$ gilts of age $90 \pm 4 \mathrm{~d}$ from a commercial farm were housed in National Engineering and Technology Research Center for Livestock and fed with diets according to NRC (2012). Experimental design: $\mathrm{L} \times \mathrm{Y}$ gilts receive $20 \mathrm{~mL}$ saline (Ctrl) or fecal suspension from Meishan gilts (FMT) and these Meishan gilts receive the same volume of saline (MS) every other day until the puberty onset. For reducing the anaerobic bacteria die, we prepared fecal slurry with $\mathrm{O}_{2}$ free saline immediately after collecting fresh feces. We then regulated the concentration of fecal slurry after counting the live microbes using optical microscopy combined with methylene blue staining [18]. All gilts were sacrificed at $24 \mathrm{~h}$ after detection of the third estrus (excluded a Meishan gilts because of its health concerns). Blood samples $(10 \mathrm{~mL})$ were collected and then centrifugate for $10 \mathrm{~min}$ at $3,500 \times \mathrm{g}$ at $4{ }^{\circ} \mathrm{C}$ to get plasma samples. Immediately after slaughter, intestinal contents from rectum were collected and placed in liquid nitrogen. We determined the weight and length of uterine horn. Uterus tissue was removed from the middle portion of the uterus and rinsed with iced cold PBS, then fixed in $4 \%$ paraformaldehyde solution for measuring uterus morphology or placed in liquid nitrogen.

Fecal microbiota transplantation (FMT) experiment The fecal suspension was prepared using the protocol previously described with minor optimization [19]. 
Briefly, fresh feces samples were obtained from Meishan gilts and immediately homogenized in sterile and $\mathrm{O}_{2}$ free saline solution. Then fecal slurry was passed through the sterilized gauze and then a $0.224-\mathrm{mm}$ stainless cell strainer to remove the particles. We used optical microscopy combined with methylene blue staining to count the live microbes in the fecal slurry. The Meishan gilts' neighbor $\mathrm{L} \times \mathrm{Y}$ gilts received sterile saline $(20 \mathrm{~mL})$ or fecal microbiota suspensions $\left(10^{7} \mathrm{CFU} / \mathrm{mL}, 20 \mathrm{~mL}\right)$ every other day from the age of $90 \mathrm{~d}$ to the age of $\mathrm{pu}$ berty onset (about $200 \mathrm{~d}$ of age).

\section{Evaluation of endometrial gland development}

We evaluated endometrial gland development by examining hematoxylin-and eosin-stained (H\&E) uterine wall sections $(10 \mu \mathrm{m})$ using computer-assisted morphometry. For each section, six areas of the endometrium from lumen to myometrium were randomly selected and measured. Then, the total area of glands within the endometrial area was measured by encircling each gland within the previously outlined endometrial area. Gland development for that section was then expressed as the ratio of gland area to endometrial area [20].

\section{Gut microbiota profiling}

The total genomic DNA of fecal bacteria (stool from the rectum) was extracted using the protocol of the $\mathrm{Re}$ peated Bead Beating Plus Column Method [21]. The integrity of DNA was assessed by agarose gel electrophoresis. The genomic DNA was used as a template for PCR amplification. Universal primers $338 \mathrm{~F}$ and 806R were used for PCR amplification of the V3-V4 hypervariable regions of $16 \mathrm{~S}$ rRNA genes (338F, $5^{\prime}$-ACTC CTACGGGAGGCAGCA-3', 806R, 5' -GGACTACHVG GGTWTCTAAT-3') [22]. The generated DNA pool was then sequenced on the Illumina HiSeq system with the sequencing strategy PE300 and then the sequencing data were analyzed using the Quantitative Insights Into Microbial Ecology software package [23].

To obtain more accurate and reliable results in subsequent bioinformatics analysis, raw data were cleansed by the in-house procedure [22]. Then paired end reads with overlap were merged to tags. The high-quality pairedend reads were combined to tags based on overlaps with FLASH [24]. The tags were clustered to OTU (Operational Taxonomic Unit) by scripts of software USEARCH(v7.0.1090, [25]). After that, they were clustered into OTU with a 97\% threshold using UPARSE, and the OTU unique representative sequences were obtained; Chimeras were filtered out by UCHIME(v4.2.40); OTU representative sequences were taxonomically classified using Ribosomal Database Project (RDP) Classifier v.2.2 trained on the database Greengene_2013_5_99 [26] with 0.6 confidence values as cutoff. The alpha diversity indices including observed species value, Chao1 value, ACE value, Shannon, and Simpson value are calculated by Mothur (v1.31.2, [27]) with the corresponding rarefaction curve was drawn by software R (v3.6.0). Phylogenetic beta diversity measures such as unweighted UniFrac distance metrics analysis and principalcomponent analysis (PCoA) were done using the QIIME 2 [23]. Functional analysis of gut microbiota was predicted by Phylogenetic Investigation of Communities by Reconstruction of Unobserved States 2 (PICRUSt2, [28]).

\section{Gut microbial metabolites}

We determined the microbial Metabolites short-chain fatty acids (SCFAs) (acetate, propionate, butyrate) in the diegesta of colon using gas chromatography with a modification of the previous method [29]. In brief, $1 \mathrm{~g}$ of the digesta samples was weighed into a 2-mL centrifuge tube with $1 \mathrm{~mL}$ of methanol added. After being vortexed for $30 \mathrm{~s}$, the sample was centrifuged $(12,000 \times g)$ at $4{ }^{\circ} \mathrm{C}$ for $10 \mathrm{~min}$. The supernatant was transferred into centrifuge tubes $(2 \mathrm{~mL})$ and mixed with $0.2 \mathrm{~mL} 25 \%$ metaphosphoric acid. After $30 \mathrm{~min}$ at $4{ }^{\circ} \mathrm{C}$, the tubes were centrifuged $(12,000 \times g)$ again at $4{ }^{\circ} \mathrm{C}$ for $10 \mathrm{~min}$. To quantify SCFAs, a calibration curve for the concentration range of $0.015-1 \mathrm{mg} / \mathrm{mL}$ was constructed. SCFAs measurements were performed following a recently published protocol [30].

Quantitative enzyme-linked immunosorbent assay (ELISA) Steroid hormones estradiol, progesterone, and insulinlike growth factor 1 (IGF-1) in plasma and tissue were quantified by ELISA (Cusabio, Wuhan, China). We collected plasma samples using EDTA as an anticoagulant and then the samples were centrifuged for $15 \mathrm{~min}$ at $1,500 \times g$ and $4{ }^{\circ} \mathrm{C}$. For tissue homogenates, $100 \mathrm{mg}$ tissue was rinsed with $1 \times \mathrm{PBS}$, homogenized in $1 \mathrm{~mL}$ of $1 \times$ PBS and stored overnight at $-20^{\circ} \mathrm{C}$. After two freezethaw cycles were performed to break the cell membranes, the homogenates were centrifuged for $5 \mathrm{~min}$ at $5,000 \times g$, and $4{ }^{\circ} \mathrm{C}$. The supernatant was removed and assayed immediately. Alternatively, aliquot and store samples at $-20^{\circ} \mathrm{C}$ or $-80^{\circ} \mathrm{C}$. Centrifuge the sample again after thawing before the assay. Avoid repeated freeze-thaw cycles. All samples were measured in 3 replicates following the recommended procedures in the instruction.

\section{Quantitative real-time PCR}

qPCR was performed with the PowerUp ${ }^{\text {тм }}$ SYBR $^{\text {тм }}$ Green Master Mix (Applied Biosystems) on a CFX384 RealTime PCR system (Bio-Rad) and changes in gene expression were calculated relative to GAPDH. RT-qPCR primer pairs are: estrogen receptor 1 gene (ESR1) 5' -AGCACCCTGAAGTCTCTGGA-3', $\quad$ 5' -TGTGCC 
TGAAGTGAGACAGG-3'; epithelial cadherin (CDH1) 5' - ATGTGCACGTATGCGACTGT-3', 5'-GGAACT TGCAATCCTGCTTC-3; forkhead box protein A2 (FOXA2) 5' -ATGCTGGGAGCGGTGAAGAT-3', 5'AGCGAGTGGCGGATGGAGTT-3'; glyceraldehyde-3phosphate dehydrogenase (GAPDH) 5'-T CGGAGTGA ACGGATTTGGC-3', 5'-TGCCGTGGGTGGAATCAT AC-3'.

\section{Hematoxylin and eosin (H\&E) staining}

Briefly, ovaries were preserved in $4 \%$ paraformaldehyde overnight at $4{ }^{\circ} \mathrm{C}$ (Sigma-Aldrich). The tissues were subsequently embedded in paraffin wax (Fisher Scientific) following immersion in a graded series of alcohols (70$100 \%)$. Embedded tissue was sectioned $(5 \mathrm{~mm})$ using a rotary microtome. We adhered these sections to microscope slides and then dried them at $56^{\circ} \mathrm{C}$ for $24 \mathrm{~h}$. Next, we passed slides through a series of the clearing agent xylene and rehydrated in a graded series of ethanol $(100 \%, 100 \%, 100 \%, 95 \%, 80 \%)$. After a brief wash in distilled water, we incubated the slides with hematoxylin solution. The sections were then washed with running tap water to remove excess hematoxylin. Then, we differentiated the sections in 1\% acid alcohol for $30 \mathrm{~s}$ and then washed them with running tap water for $1 \mathrm{~min}$. This step was followed by an incubation in the eosin counterstain, subsequent dehydration in a graded series of ethanol (80\%, 95\%, 95\%, 95\%, 100\%, 100\%, 100\%), and immersion in xylene.

\section{Untargeted metabolomics profiling}

Sample preparation. Plasma metabolite extraction was performed by mixing $40 \mu \mathrm{L}$ thawed plasma and $120 \mu \mathrm{L}$ ice-cold methanol, vortexing for $1 \mathrm{~min}, 10 \mathrm{~min}$ standing at room temperature, and keeping at $-20{ }^{\circ} \mathrm{C}$ for $2 \mathrm{~h}$. After centrifuged at $4,000 \times g$ and $4{ }^{\circ} \mathrm{C}$ for $30 \mathrm{~min}, 25 \mu \mathrm{L}$ of the supernatant was transferred into a glass vial containing $225 \mu \mathrm{L}$ of $50 \%$ methanol. Take $50 \mu \mathrm{L}$ of each sample and mix it into a quality control (QC) sample and divide all samples into 96-well plates in the order of loading and put $60 \mu \mathrm{L}$ in each well. Liver metabolite extraction was performed by mixing $25 \mathrm{mg}$ thawed liver tissue and $800 \mu \mathrm{L}$ of chilled methanol/water (1:1) solution. Add two small steel balls to each EP tube, place the sample in TissueLyser and grind. The parameter is set to $50 \mathrm{~Hz}$ for $3 \mathrm{~min}$. After grinding, remove the steel ball, place the EP tube in the $-20^{\circ} \mathrm{C}$ refrigerator for $2 \mathrm{~h}$. After centrifuged at $30,000 \times g$ and $4{ }^{\circ} \mathrm{C}$ for $15 \mathrm{~min}$, carefully take $650 \mathrm{~mL}$ of each sample into a new EP tube. Then centrifuged at $25,000 \times g$ and $4{ }^{\circ} \mathrm{C}$ for $20 \mathrm{~min}$, take $550 \mathrm{~mL}$ of each sample in a new EP tube. Take $1 \mathrm{~mL}$ of acetonitrile through the column with a positive pressure extractor, then take $1 \mathrm{~mL}$ of $100 \%$ methanol through the column at a constant rate, and finally take $1 \mathrm{~mL}$ of Milli-
Q pure water through the column at a constant rate. Draw $400 \mathrm{~mL}$ supernatant after centrifugation for each sample, transfer to the activated and equilibrated SPE column, after repeating once, discard the supernatant, add $400 \mathrm{~mL} \mathrm{100 \%}$ acetonitrile to elute, keep eluted solution in a new EP tube. Take $40 \mu \mathrm{L}$ of all samples and mix them into QC. Dispense all the samples into 96-well plates with $60 \mu \mathrm{l}$ of each sample in each well.

Metabolomics analysis. For plasma and liver tissue sample, all samples were gained by the Liquid Chromatography Mass Spectrometry (LC-MS) system followed machine orders. First, all chromatographic separations were performed using an ultra-performance liquid chromatography (UPLC) system (Waters, UK). For the reversed phase separation, plasma sample used an ACQUITY UPLC BEH C18 column $(100 \mathrm{~mm} \times 2.1 \mathrm{~mm}$, $1.7 \mu \mathrm{m}$, Waters, UK) and liver tissue sample used an ACQUITY UPLC HSS T3 column $(100 \mathrm{~mm} \times 2.1 \mathrm{~mm}$, $1.8 \mu \mathrm{m}$, Waters, UK). The column oven was maintained at $50{ }^{\circ} \mathrm{C}$. The flow rate was $0.4 \mathrm{~mL} / \mathrm{min}$ and the mobile phase comprised solvent $\mathrm{A}$ (water $+0.1 \%$ formic acid) and solvent B (acetonitrile $+0.1 \%$ formic acid). Gradient elution conditions were set: $0-2 \mathrm{~min}, 100 \%$ phase A; 2$11 \mathrm{~min}, 0$ to $100 \% \mathrm{~B} ; 11-13 \mathrm{~min}, 100 \% \mathrm{~B} ; 13-15 \mathrm{~min}, 0$ to $100 \% \mathrm{~A}$. The injection volume for each sample was $10 \mu \mathrm{L}$. A high-resolution tandem mass spectrometer Xevo G2 XS QTOF (Waters, UK) was used to detect metabolites eluted form the column. The Q-TOF was operated in both positive and negative ion modes. For positive ion mode, the capillary and sampling cone voltages were set at $3.0 \mathrm{kV}$ and $40.0 \mathrm{~V}$, respectively. For negative ion mode, the capillary and sampling cone voltages were set at $2.0 \mathrm{kV}$ and $40.0 \mathrm{~V}$, respectively. The mass spectrometry data were gained in Centroid MSE mode. The TOF mass range was from 50 to $1200 \mathrm{Da}$ and the scan time was $0.2 \mathrm{~s}$. For the MS/MS detection, all precursors were fragmented using $20-40 \mathrm{eV}$, and the scan time was $0.2 \mathrm{~s}$. During the acquisition, the LE signal was gained every $3 \mathrm{~s}$ to calibrate the mass accuracy. In order to test the stability of the LC-MS during the whole acquisition, a quality control sample (Pool of all samples) was gained after every 10 samples. Peak extraction is mainly achieved through the commercial software Progenesis QI (version 2.2), including peak alignment, peak extraction, normalization, deconvolution, and compound identification. Based on QC sample information, local polynomial regression fitting signal correction (Quality control-based robust LOESS signal correction, QCRSC) is performed on the real sample signal [31].

\section{Statistical analysis}

Results were presented as mean \pm SEM. Experimental data were analyzed by one-way analysis of variance tests, followed by Fisher's least significant difference and the 
Duncan multiple comparison test with GraphPad 8.0 software. Significance was presented as $* P<0.05$, and $\because * P<0.01$, whereas $P$ values between 0.05 and 0.10 were considered as indicative of a trend.

\section{Results}

Meishan gilts have distinct uterine characters and gut microbiota compared with LxY gilts

Under the same housing and feeding conditions, Meishan gilts have larger $(P<0.001)$ endometrial gland area normaled to the endometrial area (Fig. 1a-b) and longer $(P<0.01$ for left uterine horn and $P<0.001$ for right uterine horn) uterine horn length (Fig. 1c) but lighter $(P<0.05)$ uterine horn weight (Fig. 1d) compared with $\mathrm{L} \times \mathrm{Y}$ gilts at the age of third estrus. The beta diversity of gut microbiota showed by PCoA showed obvious difference between Meishan gilts and LxY gilts (Fig. 1e). Meishan gilts had superior alpha diversity indices including observed species $(P<0.01)$, Chao1 $(P<0.01)$, ACE $(P<0.01)$, Shannon $(P<0.01)$ and Simpson $(P<0.001)$ compared with $\mathrm{L} \times \mathrm{Y}$ gilts (Fig. 1f). The alpha diversity index Good coverage suggested that all the sequencing data were of high quality and could cover the most species. Further taxon analysis showed that Meishan gilts' gut harbored more bacteria of phyla Actinobacteria $(P<$ $0.01)$, Firmicutes $(P<0.01)$, Fibrobacteres $(P<0.001)$, Lentisphaerae $(P<0.05)$, and Synergistetes $(P<0.01)$ but fewer bacteria of phyla Bacteroidetes $(P<0.001)$, and WPS-2 $(P<0.01)$ compared with $\mathrm{L} \times \mathrm{Y}$ gilts (Supplemental Figure S1a). At genus level, Meishan gilts' gut harbored more Bacteroides $(P<0.01)$, Bifidobacterium $(P<$ $0.001)$, Fibrobacter $(P<0.001)$, Oscillospira $(P<0.05)$, $\operatorname{SMB53}(P<0.05)$, and Turicibacter $(P<0.05)$ but less Anaerovibrio $(P<0.05)$, Bulleidia $(P<0.01)$, Butyricicoccus $(P<0.01)$, Coprococcus $(P<0.05)$, Dorea $(P<0.05)$, Gemmiger $(P<0.05)$, Lachnospira $(P<0.01)$, Pseudobutyrivibrio $(P<0.01)$, and Streptococcus $(P<0.05)$ compared with $\mathrm{L} \times \mathrm{Y}$ gilts (Supplemental Figure S1b).

\section{FMT shifted gut microbiota and increased the endometrial gland area in recipient LxY gilts}

To explore the association between the gut microbiota and uterine development, we transplanted the fecal microbiota of Meishan gilts to $\mathrm{L} \times \mathrm{Y}$ gilts. PCoA showed that FMT distinctly shifted the gut microbiota composition of the recipient $\mathrm{L} \times \mathrm{Y}$ gilts and made it closed to that of Meishan gilts (Fig. 2a). The top two principalcomponent (PC1 and PC2) suggested that FMT explained $60.49 \%$ of the variation (Fig. 2a). FMT recipient gilts had superior alpha diversity indices including observed species $(P<0.01)$, Chao1 $(P<0.01)$, ACE $(P<$ $0.05)$, Shannon $(P<0.05)$ and Simpson $(P<0.01)$ compared with control L $\times$ Y gilts (Fig. 2b). The alpha diversity index Good coverage suggested that all the sequencing data were of high quality and could cover the most species. Further taxon analysis showed that FMT recipient gilts' gut harbored more bacteria of phyla Firmicutes $(P<0.001)$, Fibrobacteres $(P<0.001)$, but fewer bacteria of phyla Bacteroidetes $(P<0.001)$ compared with control L $\times$ Y gilts (Supplemental Figure S2a). At genus level, FMT recipient gilts' gut harbored more Bifidobacterium $(P<0.01)$ and Fibrobacter $(P<0.01)$ compared with control $\mathrm{L} \times \mathrm{Y}$ gilts (Supplemental Figure $\mathrm{S} 2 \mathrm{~b})$. Morphological analysis(H\&E) of uterine showed that FMT recipient $\mathrm{L} \times \mathrm{Y}$ gilts had increased $(P<0.01)$ endometrial gland area (Fig. 3c-d). FMT had no effect on the uterine horn length (Fig. 3e) but had a trend ( $P=0.069$ for left uterine and $P=0.067$ for right uterine) to reduce the uterine horn weight (Fig. 3f).

\section{Untargeted metabolomics revealed metabolism shift induced by FMT in liver and plasma}

To link the shifted gut microbiota and increased endometrial gland area, we determined the metabolites profiles of liver and plasma using untargeted metabolomics. Partial least squares discriminant analysis (PLS-DA) showed that the detected metabolites of both pos and neg mode were obviously shifted by FMT in liver (Fig. 3a) and plasma (Fig. 3b). The volcano plots showed the significantly differential metabolites (red dot) under both pos and neg mode in liver (Fig. 3c) and plasma (Fig. 3d). We further conducted KEGG pathway enrichment analysis for the different metabolites in both liver and plasma. For liver, the FMT-induced differential metabolites enriched in pathways of steroid hormone biosynthesis, steroid biosynthesis, ovarian steroidogenesis, linoleic acid metabolism, folate biosynthesis, and biosynthesis of unsaturated fatty acids (Fig. 3e). And for plasma, the FMT-induced differential metabolites enriched in pathways of steroid hormone biosynthesis, primary bile acid biosynthesis, fatty acid metabolism, fatty acid degradation, estrogen signaling pathway, butanoate metabolism, and alpha-Linolenic acid metabolism (Fig. 3f). Noteworthily, steroid hormone biosynthesis pathway was enriched by FMT-induced differential metabolites from both liver and plasma, which indicated that the circulating steroid hormone profile has been shifted by FMT.

\section{FMT shifted endometrial gland development-related hormones in recipient LxY gilts}

Given that FMT shifted the circulating steroid hormone profile, we then determined the concentration of steroid hormones estradiol and progesterone, and IGF-1 in plasma. FMT increased plasma estradiol concentration on 132 $(P<0.05), 153(P<0.05), 174(P<0.05)$, and $195(P<0.05) \mathrm{d}$ of age (Fig. 4a), but decreased plasma progesterone concentration on $111(P<0.05), 132(P=$ 


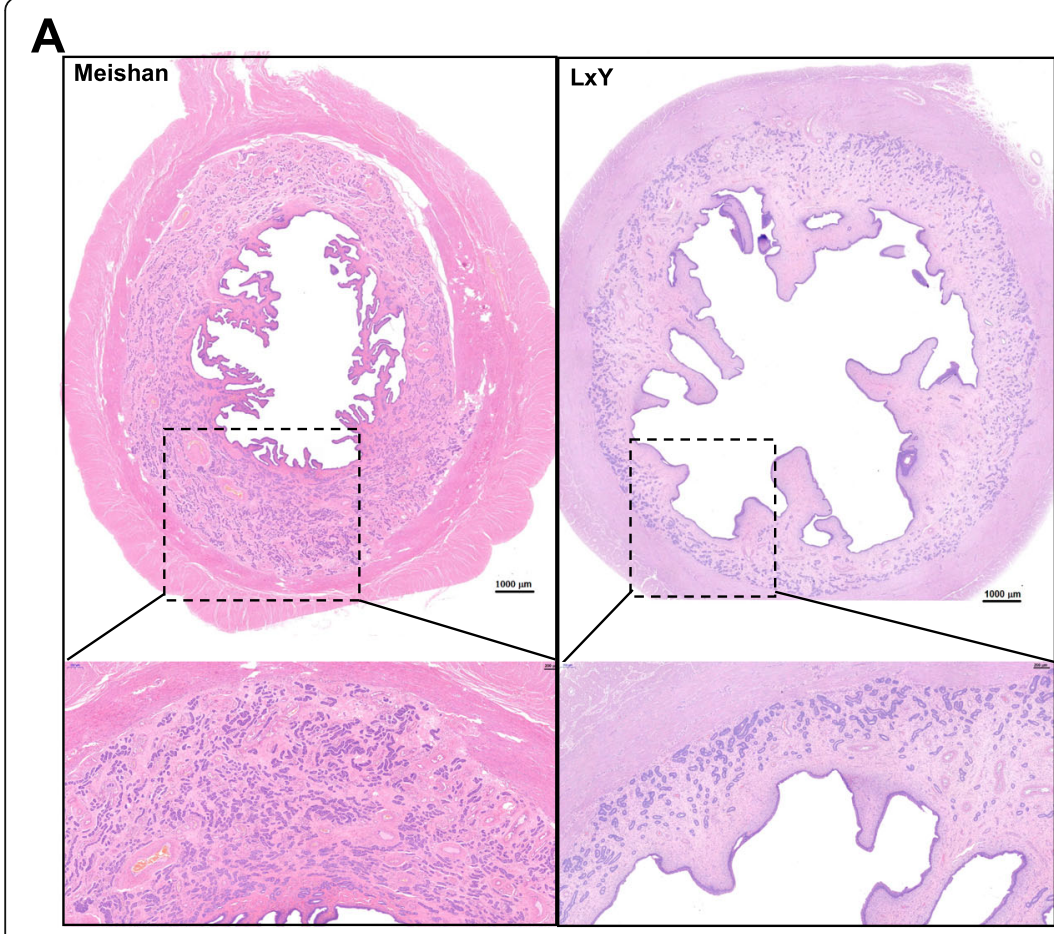

\section{B}
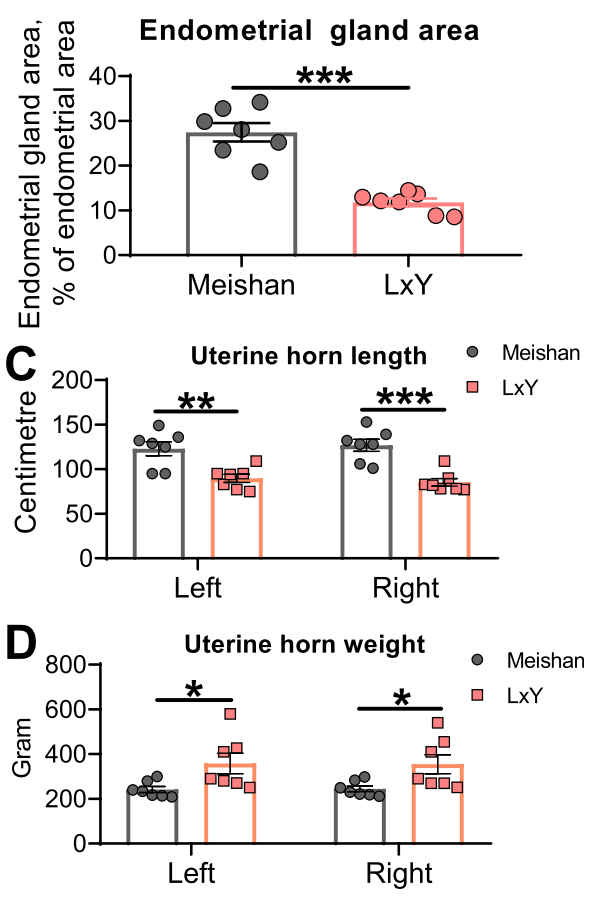

$\mathbf{E}$

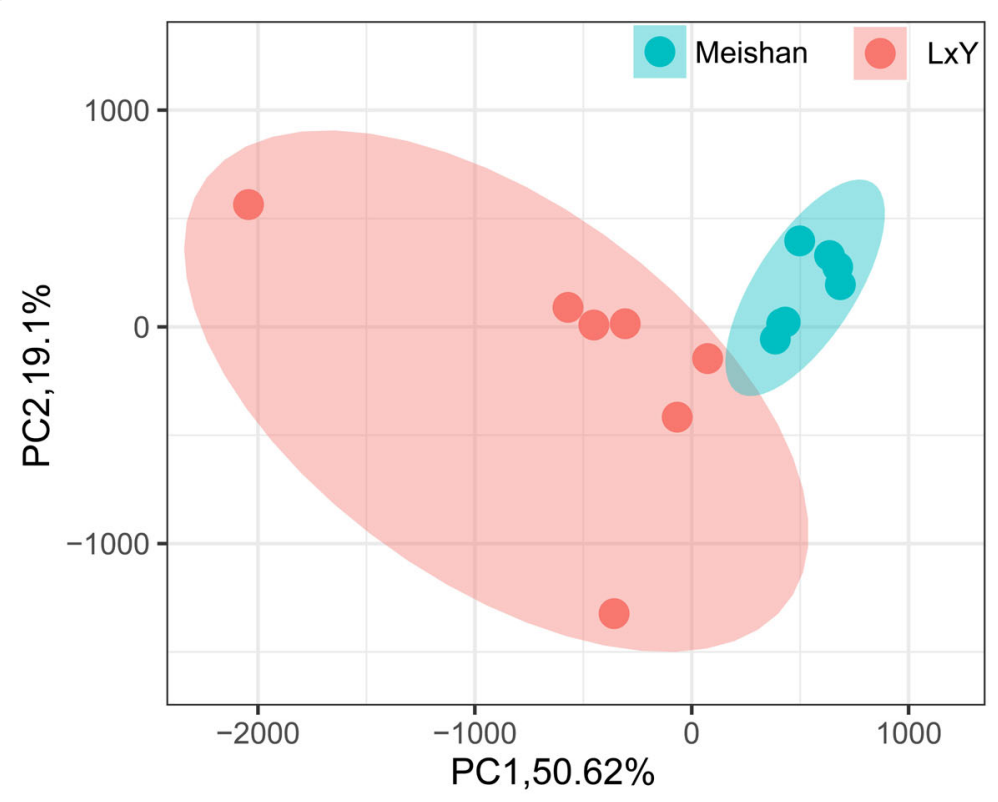

$\mathbf{F}$
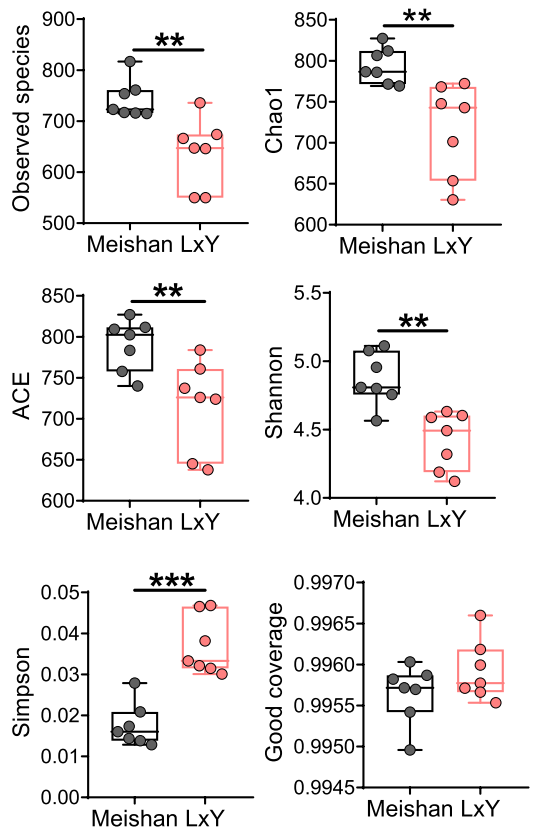

Fig. 1 Meishan gilts have distinct uterine characters and gut microbiota compared with LXY gilts. a Representative H\&E-stained uterine sections. Scale bar, 1,000 $\mathrm{mm}$ and $200 \mu \mathrm{m}$. b Endometrial gland area normalized to endometrial area. Length (c) and weigh (d) of Uterine horn. e PCoA based on weighted UniFrac distance show a distinct fecal microbial composition inter-groups. Each data point represents a sample from a distinct sow projected onto the first two principal coordinates (percent variation explained by each PCo is shown in parentheses). $\mathbf{f}$ Species diversity analysis of gut microbiota with alpha diversity indices including Observed species, Chao1, ACE, Shannon, Simpson and Good coverage. ${ }^{*} P<0.05,{ }^{* *} P<0.01,{ }^{* * *} P<0.001$, data were shown as means $\pm \mathrm{SEM} ; n=7$ 


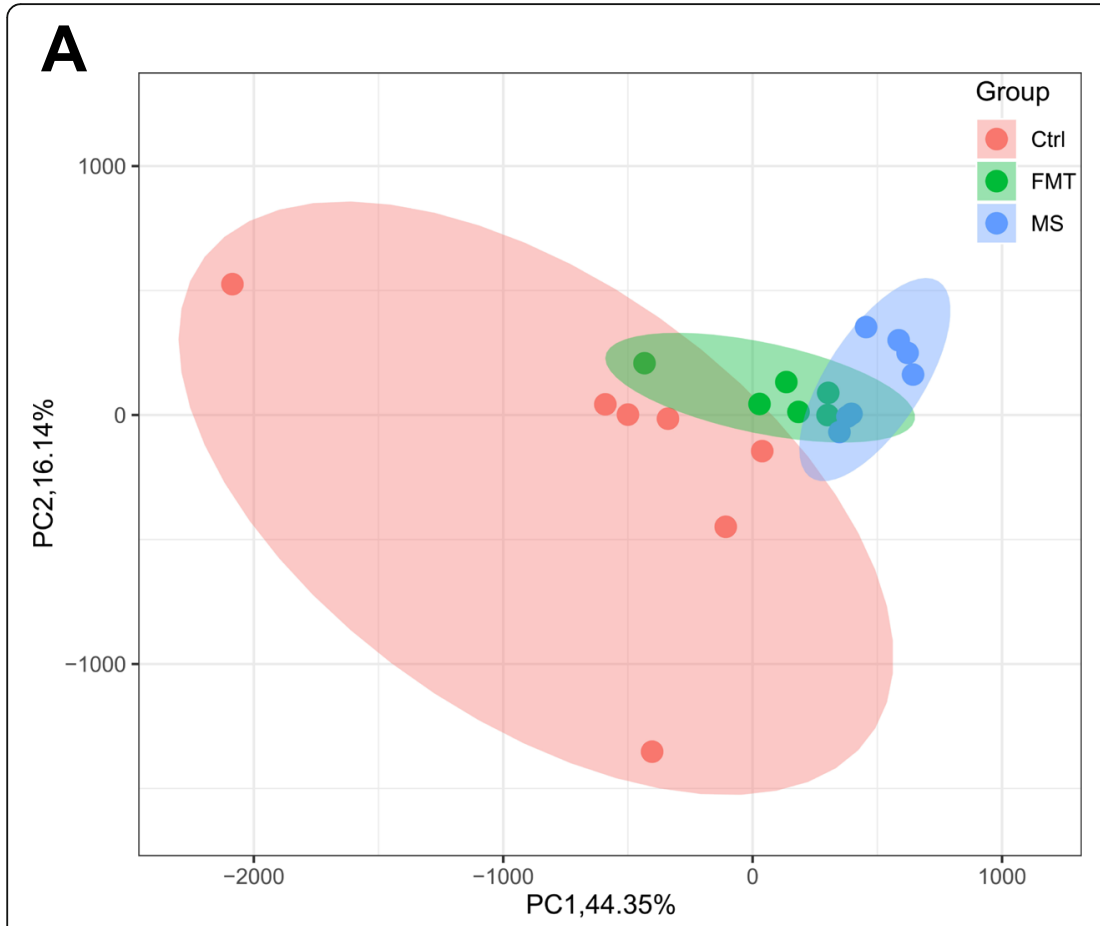

B

C

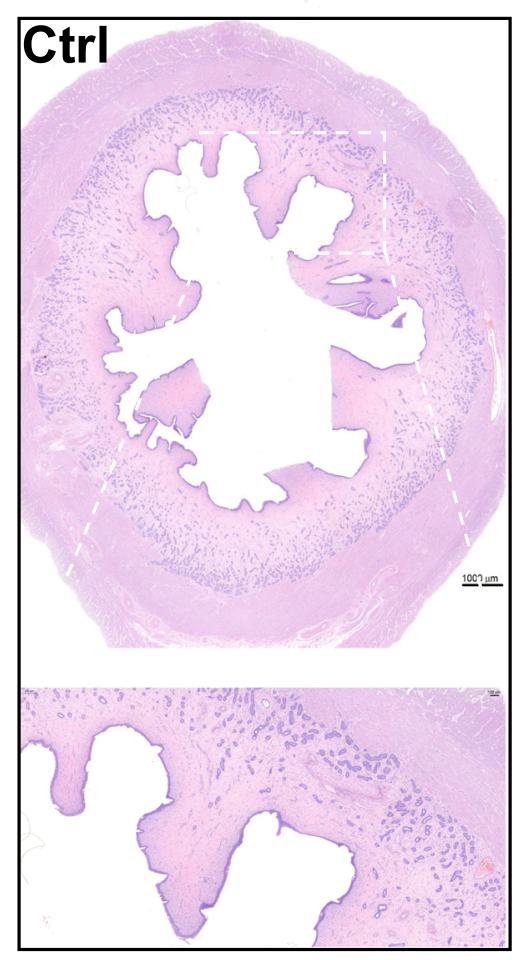

FMT

1000 um

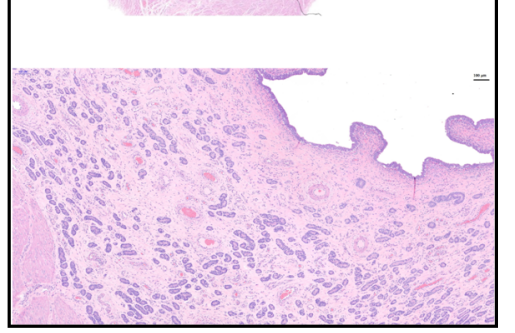

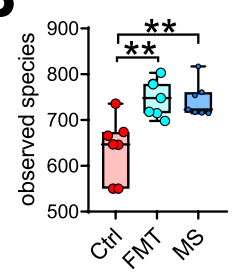
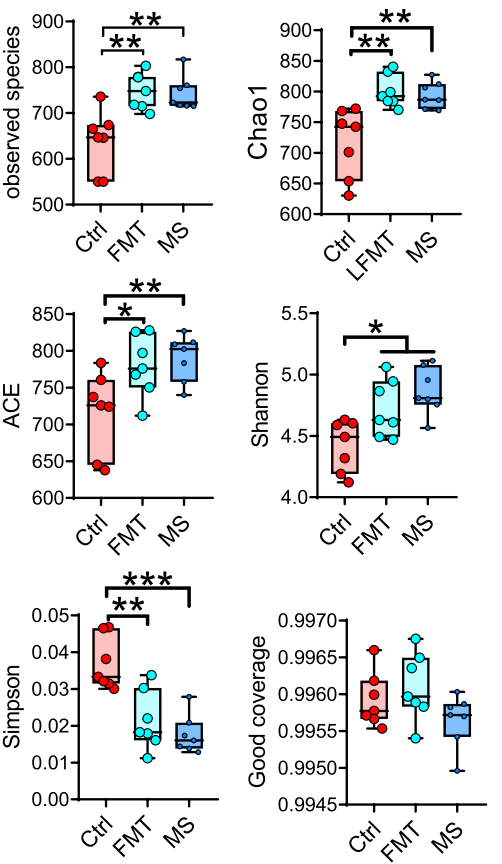

D

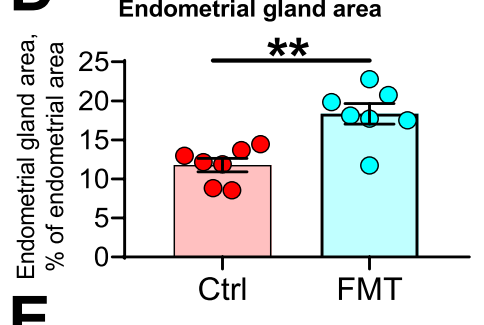

$\mathbf{E}$

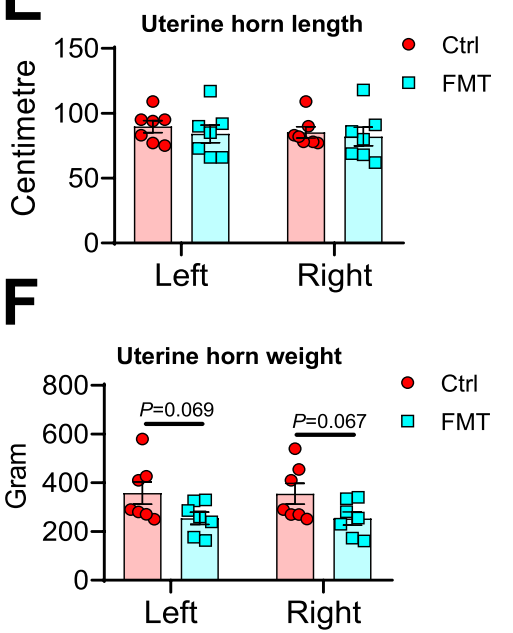

Fig. 2 FMT shifted gut microbiota and increased the endometrial gland area in recipient $L X Y$ gilts. a PCoA based on weighted UniFrac distance show a distinct fecal microbial composition intergroups. Each data point represents a sample from a distinct sow projected onto the first two principal coordinates (percent variation explained by each PCo is shown in parentheses). b Species diversity analysis of gut microbiota with alpha diversity indices including Observed species, Chao1, ACE, Shannon, Simpson and Good coverage. c Representative H\&E-stained uterine sections. Scale bar, 1,000 $\mu \mathrm{m}$ and $200 \mu \mathrm{m}$. d Endometrial gland area normalized to endometrial area. Length (e) and weigh (f) of Uterine horn. ${ }^{*} P<0.05$, ${ }^{* *} P<0.01,{ }^{* * *} P<0.001$, data were shown as means $\pm \mathrm{SEM} ; n=7$ 


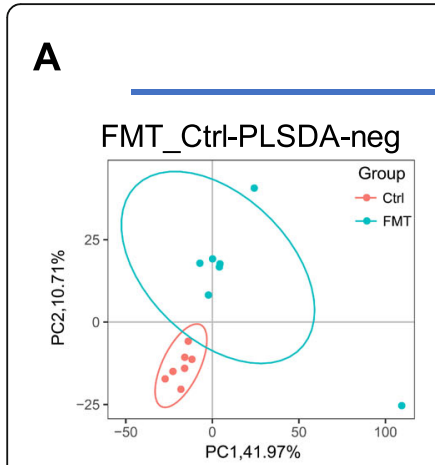

Liver
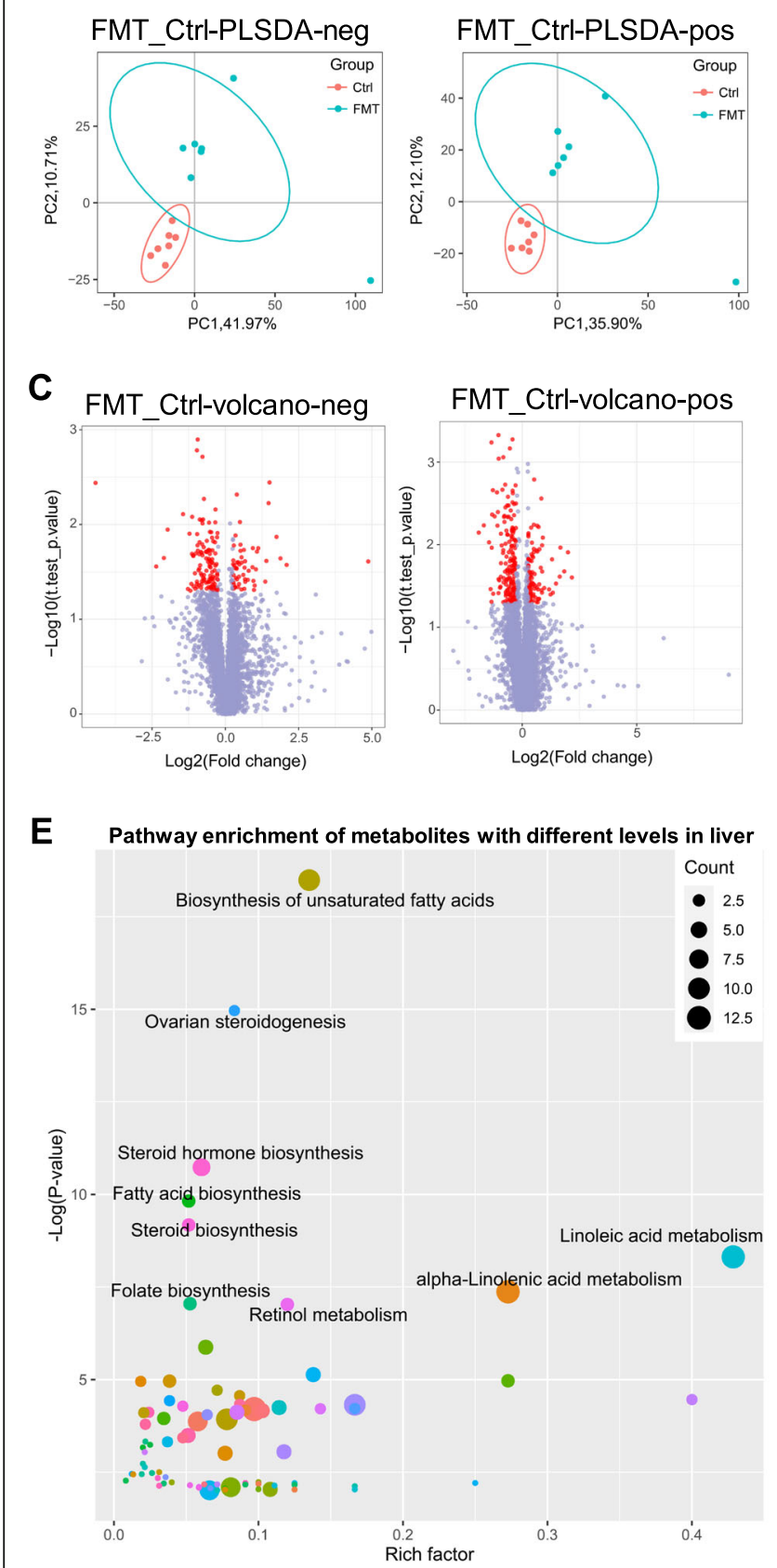

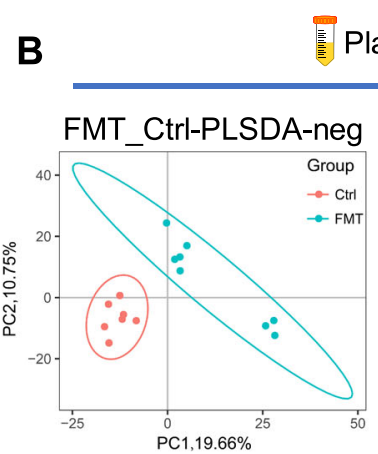

Plasma
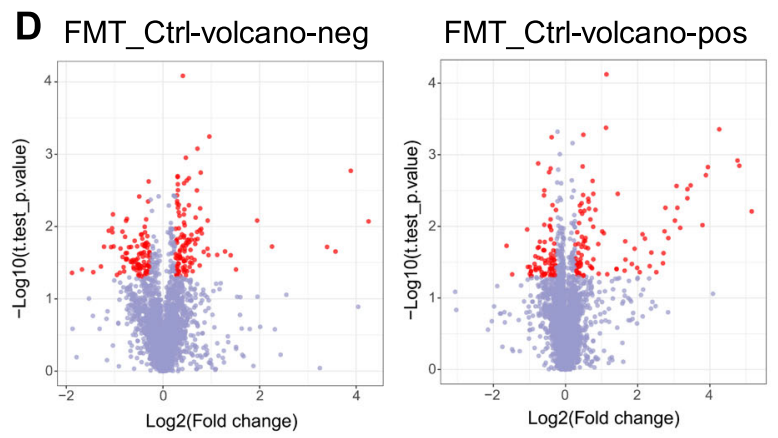

F Pathway enrichment of metabolites with different levels in plasma
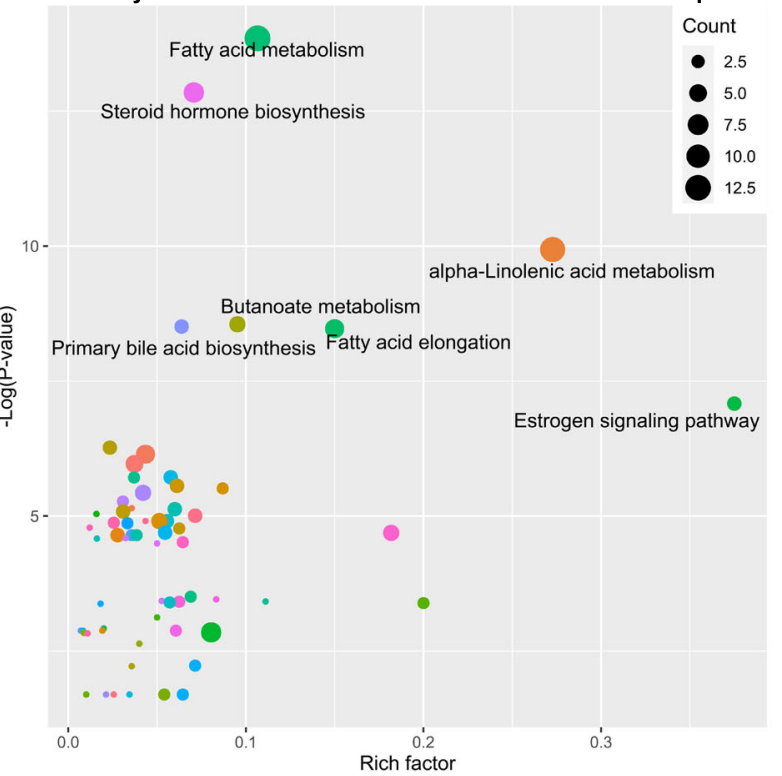

Fig. 3 FMT shifted the metabolite profiles of liver and plasma in recipient LXY gilts. PLS-DA of the detected metabolites of both pos and neg mode in liver (a) and plasma (b). The volcano plots of the significantly differential metabolites (red dot) under both pos and neg mode in liver (c) and plasma (d). KEGG pathway enrichment analysis for the different metabolites in both liver (e) and plasma (f). $n=7$

$0.057)$, and $153(P<0.05) \mathrm{d}$ of age (Fig. 4b). Estradiol and progesterone can regulate of IGF-1 gene expression in pig uterus [32]. We therefore determined the IGF-1 concentration in plasma and uterine tissue. The results showed that FMT increased IGF-1 on $111(P<0.05)$, 132 $(P<0.05), 153(P=0.07), 174(P<0.05)$, and 195 $(P<$
$0.01) \mathrm{d}$ of age (Fig. 4c), and in uterine tissue $(P<0.05)$ (Fig. 4f). The enhanced secretion of estradiol $(P<0.01)$ and progesterone $(P<0.001)$ were determined in ovary tissue (Fig. 4d-e). Correspondingly, FMT elevated $(P<$ $0.01)$ the mRNA expression level of estrogen receptor 1 gene (Esr1) (Fig. 4g). Besides, FMT elevated the mRNA 


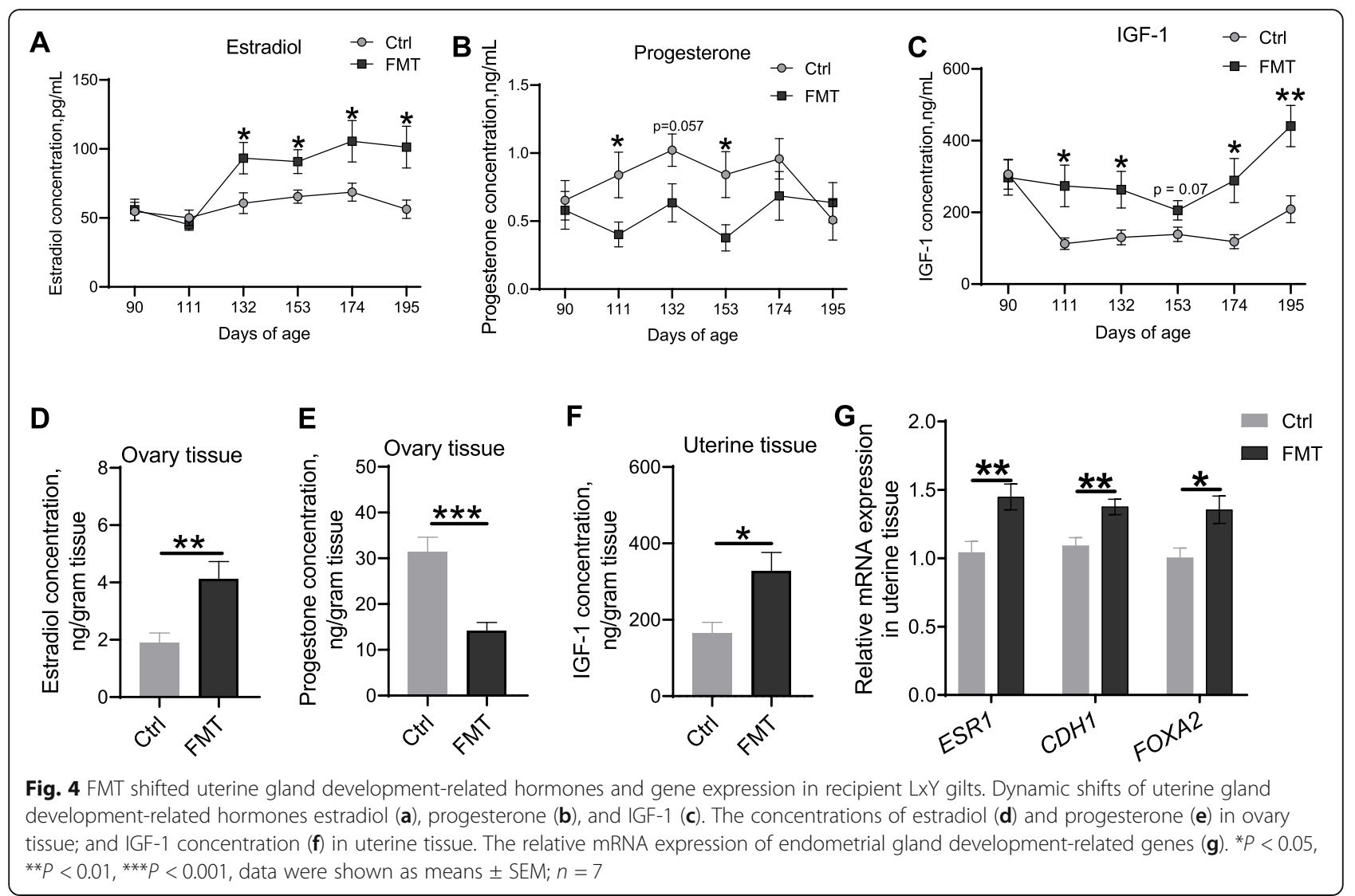

expression level of uterine development -related epithelial cadherin $(C D H 1)(P<0.01)$ and forkhead box protein A2 $(F O X A 2)(P<0.05)$ (Fig. $4 \mathrm{~g})$.

\section{FMT promoted carbohydrate metabolism and digestive} system functions of gut microbiota in recipient LxY gilts To explore the underlying mechanism by which the shifted gut microbiota contributes to the endometrial gland development, we determined the function shift of gut microbiota using PICRUSt2. The results suggested that FMT enriched KEGG functions of carbohydrate metabolism $(P<0.01)$ and digestive system $(P<0.05)$ in gut microbiota of recipient $\mathrm{L} \times \mathrm{Y}$ gilts (Fig. $5 \mathrm{a}$ ). This enrichment effect of FMT was confirmed by the increased concentrations of SCFAs propionate $(P<0.01)$ and butyrate $(P<0.05)$ but not acetate $($ Fig. 5 b-d).

\section{FMT-shifted gut microbes were correlated to the indices of uterine development}

We finally determined the correlation between FMTshifted gut microbes and uterine development-related indices using spearman's rank correlation coefficient and significance test. The results in Fig. 6 showed that Lentisphaerae $(P<0.05)$, Bifidobacterium $(P<0.05)$, and Fibro$\operatorname{bacter}(P<0.05)$ were positively correlated to endometrial gland area. Firmicutes $\left(\begin{array}{ll}P & <0.01\end{array}\right)$ and
Fibrobacter $(P<0.05)$, were positively correlated to estradiol concentration; Bacteroidetes was significantly negatively correlated to estradiol $(P<0.01)$. For IGF-1, Bacteroidetes was negatively correlated to IGF-1 concentrations $(P<0.01)$; Fibrobacteres $(P<0.05)$, Firmicutes $(P<$ $0.001)$, Bifidobacterium $(P<0.05)$, and Fibrobacter $(P<$ $0.05)$ were positively correlated to IGF-1 concentration. For progesterone, Bacteroidetes was positively correlated to progesterone concentration $(P<0.01)$; Fibrobacteres $(P<0.001)$, Firmicutes $(P<0.01)$, Bifidobacterium $(P<$ $0.01)$, and Fibrobacter $(P<0.01)$ were negatively correlated to progesterone concentration.

\section{Discussion}

Porcine uterine development plays a crucial role in determining sows' prolificacy $[4,5]$. Earlier evidences suggested that porcine uterine development occurs in an ovary-independent manner before, and in an ovarydependent manner after, $60 \mathrm{~d}$ of age $[4,5]$. Before $60 \mathrm{~d}$ of age, Christenson et al. found that Meishan gilts achieved a superior endometrial gland development than white crossbred gilts [9]. Here, we further compared the uterine development between Meishan gilts and $\mathrm{L} \times \mathrm{Y}$ gilts during ovary-dependent development period (after $60 \mathrm{~d}$ of age), and found that Meishan gilts have increased endometrial gland area and uterine horn length than 

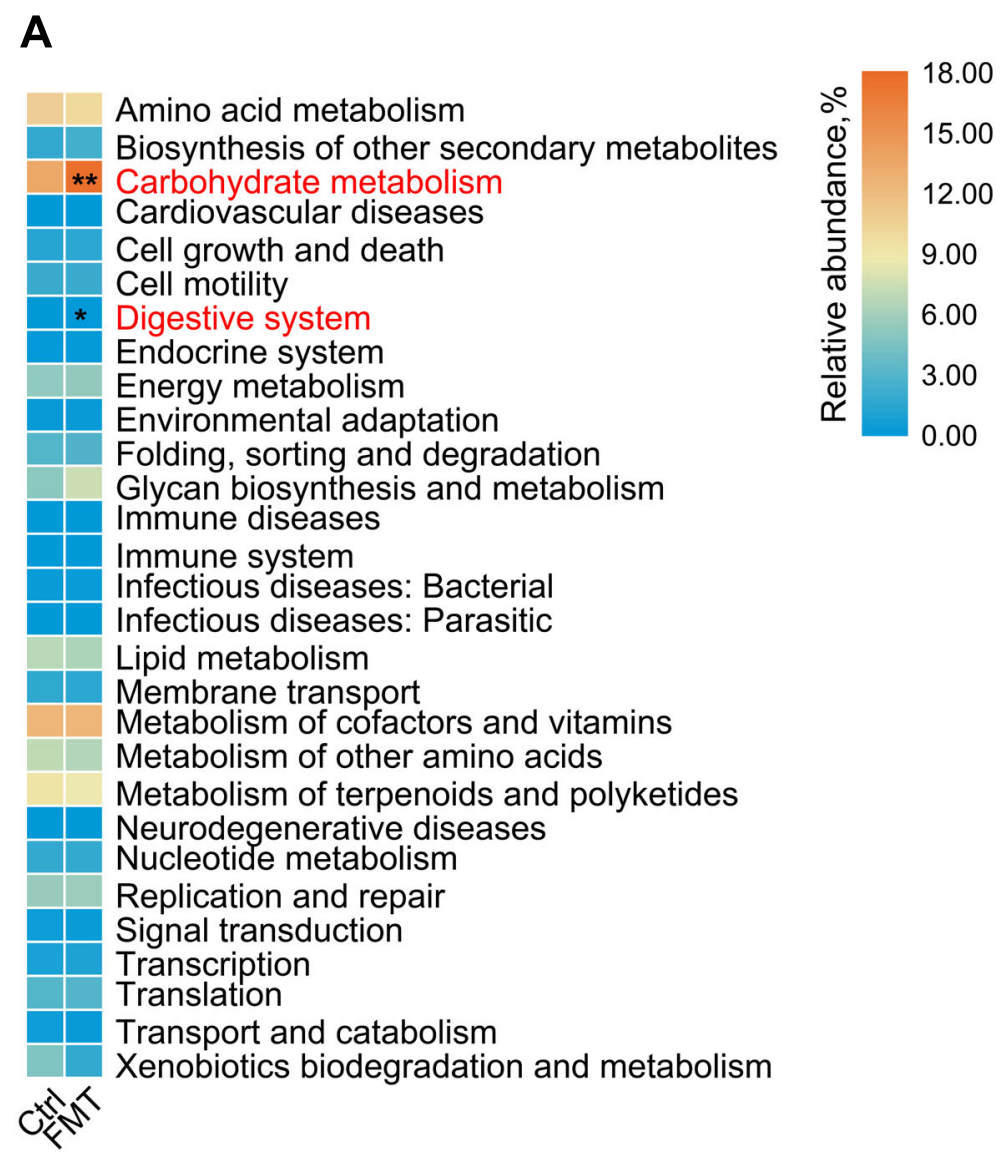

B
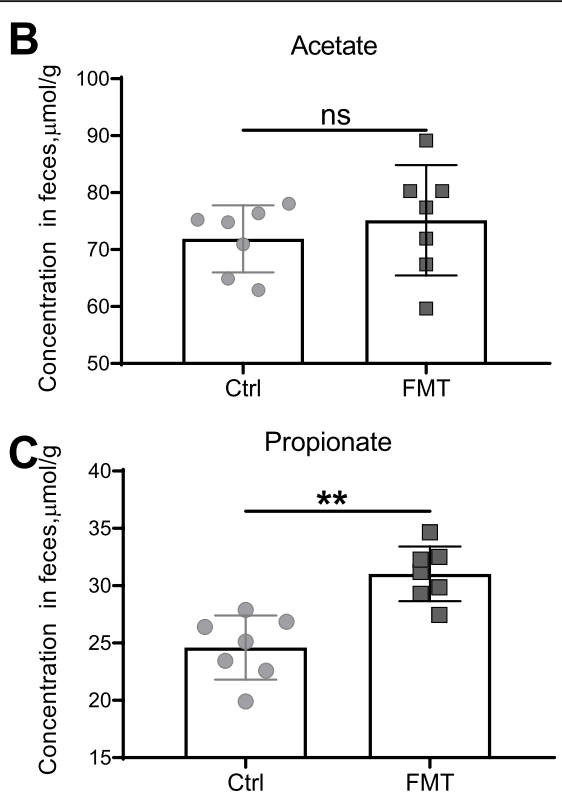

D

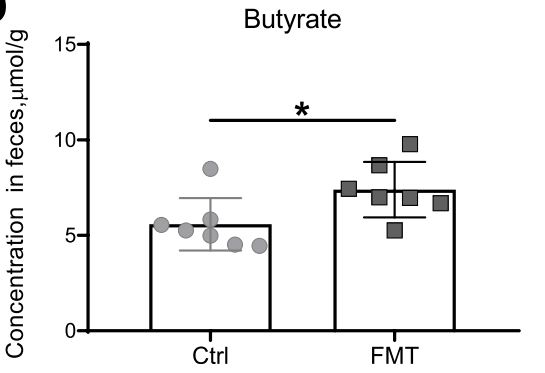

Fig. 5 FMT promoted carbohydrate metabolism and digestive system functions of gut microbiota in recipient $L \times Y$ gilts. KEGG functions of gut microbiota (a) and the corresponding concentrations of SCFAs (b). The differential KEGG function was indicated in red. ${ }^{*} P<0.05,{ }^{*} P<0.01$, data were shown as means $\pm \mathrm{SEM} ; n=7$

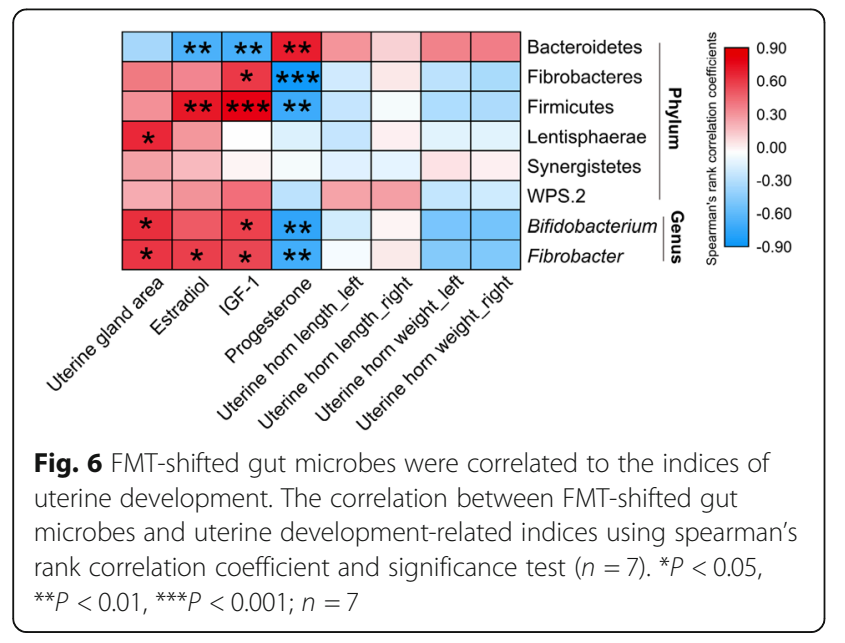

$\mathrm{L} \times \mathrm{Y}$ gilts. Thus, the superior uterine development of Meishan gilts than white crossbred $\mathrm{L} \times \mathrm{Y}$ gilts lasted from neonate to mature. In ovary-dependent development period, ovary-derived hormones estradiol and progesterone play crucial roles in endometrial glands development [5]. Recent evidences suggest that gut microbiota plays a role in the regulation of ovarian hormones estradiol and progesterone [12, 33, 34]. We found that Meishan gilts had a distinctive structure of gut microbiota and superior alpha diversity including observed species, Chao1, ACE, Shannon and Simpson compared with $\mathrm{L} \times \mathrm{Y}$ gilts under the same housing and feeding conditions. These conservative differences of gut microbiota between Meishan gilts and $\mathrm{L} \times \mathrm{Y}$ gilts indicated that host genetics can shape gut microbiota, which had been also reported in human [35]. Compared well with our results, Yuan et al. reported patients with endometriosis, a chronic and estrogen-dependent disease characterized by endometrial glands and stroma outside the uterus, had a disordered gut microbiota of reduced alpha diversity [36]. These increased alpha diversity indices had 
been also determined in Meishan sows whose reproductive performance were promoted by dietary crude fiber supplementation [10]. Further taxon analysis showed that Meishan gilts' gut harbored more bacteria of phyla Actinobacteria Firmicutes, Fibrobacteres, Lentisphaerae, and Synergistetes but fewer bacteria of phyla Bacteroidetes and WPS- 2 compared with $\mathrm{L} \times \mathrm{Y}$ gilts. Yang et al. also reported a similar difference of gut microbiota between Meishan sows and Duroc sows except Bacteroidetes [37]. These inconsistent results may because of the detect method (real-time PCR analysis vs. 16S rDNA high-throughput sequencing) or animal conditions (age etc.). Consistent with our results, Jiang et al. also determined an increased Bacteroidetes in fertility-improved Meishan sows by dietary crude fiber supplementation [10]. Among the changed bacteria at genus levels, the increased Fibrobacter, but decreased Streptococcus in Meishan gilts were comparable to the earlier reports $[10$, 38]. Taken together, our data firstly suggested that sexually mature Meishan gilts achieved superior endometrial gland area and uterine horn length than $\mathrm{L} \times \mathrm{Y}$ gilts, which may be regulated at least partly by their distinctive gut microbiota.

We then explored the association between gut microbiota and uterine development by transplanting the fecal microbiota of Meishan gilts to $\mathrm{L} \times \mathrm{Y}$ gilts. As reported in other FMT experiment [39, 40], FMT had a lasted shifteffect on the gut microbiota of recipient $\mathrm{L} \times \mathrm{Y}$ gilts and made them similar to that of the donor Meishan gilts. Interestingly, FMT from Meishan gilts increased endometrial gland area in recipient $\mathrm{L} \times \mathrm{Y}$ gilts. To further understand the association between the shifted gut microbiota and increased endometrial gland area, we profiled the metabolites of liver and plasma and found that FMT markedly shifted the metabolite profiles of both liver and plasma, and these FMT-induced differential metabolites from both liver and plasma enriched in steroid hormone biosynthesis pathway. Compared well with the results of metabolomics, we found that FMT increased estradiol concentrations, but decreased progesterone concentration in plasma during the trial dynamically. Ovary-derived steroid hormones of estradiol and progesterone play crucial roles in endometrial glands development [7]. Estradiol is a primary mitogen for uterine epithelium and can induce proliferation of both uterine luminal epithelium and glandular epithelium [7]. Progesterone reversely inhibit estradiol actions in stimulation of uterine epithelial proliferation through epithelial progesterone receptors [7, 13]. Therefore, FMT induced an increase of plasma estradiol concentrations, but a decrease of plasma progesterone concentration during our trial dynamically may account for its effect on promoting endometrial gland area. The elevated estradiol concentration but decreased progestogen in ovary tissue showed that FMT could regulate steroid hormones synthesis of ovary. Besides, estradiol and progestogen affect endometrial IGF-I expression and secretion [32]. In our study, FMT elevated IGF-1 concentration, and increased expression of ESR1 gene in uterine tissue, suggesting that gut microbiota affect endometrial IGF-I expression and secretion by regulating steroid hormones estradiol and progestogen. CDH1 and FOXA2 are essential for endometrial gland differentiation and development [41, 42]. The elevated expression of both $C D H 1$ and FOXA2 genes further confirmed that FMT promoted endometrial gland differentiation and development in recipient $\mathrm{L} \times \mathrm{Y}$ gilts. Steroid hormones regulate gene expression of $C D H 1$ during the peri-implantation period of pregnancy in pigs [43]. In ovariectomized mice, estradiol suppressed Foxa2 mRNA expression at $48 \mathrm{~h}$ but not $4 \mathrm{~h}$ and $24 \mathrm{~h}$ after treatment [44]. This suppression effect of estradiol was sped up by co-treatment with progestogen [44]. However, the role of steroid hormones on the expression of $\mathrm{CDH} 1$ and FOXA2 genes during pre-puberty gilts remain unknown. Overall, FMT shifted the steroid hormones profiles, which may contribute to its effect on promoting endometrial gland development.

Given that gut microbiota gets involved in regulation of ovarian hormones estradiol and progesterone $[12,33,34]$, we then identified the FMT-shifted bacteria. FMT increased Firmicutes, Fibrobacteres, Bifidobacterium, and Fibrobacter, but deceased Bacteroidetes. Nuriel-Ohayon et al. found that Bifidobacterium abundance increased in the gut during pregnancy in women and mice, and progesterone supplementation increases Bifidobacterium abundance in mice and in vitro [12]. Consistently, these FMTshifted bacteria along with increased estradiol and progesterone had been also determined in Meishan sows whose reproductive performance had been promoted by dietary crude fiber supplementation [10], which suggested the potential interaction between gut microbiota and progesterone. More directly, gut microbiota gets involved in enterohepatic circulation of estradiol by encoding $\beta$-glucuronidase, which plays an important role in cleaving conjugated estradiol [38, 45]. The gut microbiome includes an 'estrobolome' that the aggregate of enteric bacterial genes whose products can cleave conjugated estradiol [45]. Notably, these FMT-shifted bacteria belong to the estrobolome. Taken together, FMT-shifted gut microbiota may promoted endometrial gland area by regulating steroid hormones. Furthermore, We found that FMT enriched KEGG functions of carbohydrate metabolism and digestive system. These function shifts of gut microbiota compared well with the increased SCFAsproducing bacteria, including Fibrobacter. In addition, 
these enrichment effects of FMT was confirmed by the increased concentrations of fecal SCFAs, including propionate and butyrate but not acetate. Although there is no direct evidence of SCFAs regulating endometrial gland development, the elevated fecal SCFAs get well along with the hyper-prolificacy of sows had been reported in Meishan sows and crossed white sows $[10,38,46]$. Recently studies indicated that these elevated SCFAs may promote sows' prolificacy indirectly. Administration of SCFAs to mice alleviate stress-induced brain-gut axis alterations [47]. KimuraTodani et al. also reported that acetate exerted an anxiolytic effect on the host [48]. However, we haven't observed a significant increase of acetate concentration in distal colon, which may because of the acetate could be absorbed in colon [49]. In modern pig production system, sows suffer from postpartum stress even anxiety, which results in decreased maternal performance of lactating sows and increased preweaning piglet mortality $[50,51]$. Given that the number of pigs weaned per sow per year is used to determine sows' prolificacy [52], gut microbial metabolites SCFAs may contribute to the hyper-prolificacy of sows by ameliorating sows' postpartum stress. Besides, the significant correlation between FMT-shifted gut microbes and uterine development-related indices further supports our hypotheses that gut microbiota contribute to endometrial gland development during the ovary-dependent period.

\section{Conclusion}

In conclusion, this study presented that sexually mature Meishan gilts achieved a superior uterine development than $\mathrm{L} \times \mathrm{Y}$ gilts under the same housing and feeding conditions. Meanwhile, Meishan gilts harbored a distinctive gut microbiota that superior in alpha diversity when compared with $\mathrm{L} \times \mathrm{Y}$ gilts. Transplanting fecal microbiota from Meishan gilts made the gut microbiota of recipient $\mathrm{L} \times \mathrm{Y}$ gilts similar to that of the donors lastingly and promote endometrial gland development, simultaneously. The FMT-induced profiler shifts of steroid hormones which potentially driven by gut microbiota contribute to the promotion of endometrial gland development. Therefore, our data suggests that gut microbiota contribute to the endometrial gland development during the ovary-dependent period.

\footnotetext{
Abbreviations

SCFAs: Short chain fatty acids; ELISA: Enzyme-linked immunosorbent assay; IGF-1: Insulin-like growth factor 1; EDTA: Ethylenediaminetetraacetic acid; ESR1: Estrogen receptor 1 gene; $\mathrm{CDH1}$ : Cadherin-1; FOXA2: Forkhead box protein A2; H\&E: Hematoxylin and eosin; LC-MS: Liquid chromatography mass spectrometry; PICRUSt2: Phylogenetic Investigation of Communities by Reconstruction of Unobserved States 2; OTU: Operational taxonomic units
}

\section{Supplementary Information}

The online version contains supplementary material available at https://doi. org/10.1186/s40104-021-00578-y.

Additional file 1: Supplemental Table S1. Ingredient composition and chemical analysis of the basal diet for tilapia.

Additional file 2: Supplemental Figure S1. Relative abundance of gut microbes in Meishan and LXY gilts.

Additional file 3: Supplemental Figure S2. FMT shifted the composition of gut microbiota in recipient LXY gilts.

\section{Acknowledgements}

We thank Yan lab's members for helpful discussions and critical reading of the manuscript.

\section{Authors' contributions}

B. X., L.M., and X.Y. designed the research. B.X., Y.Y., W.Q., Y. T., S.Z., and J. H. performed the research. B.X., Y.Y., W.Q., Y. T., S.Z., and X.Y. analyzed the data. B.X., and X.Y. wrote the paper with the help of all authors. All authors read and approved the final version of the manuscript.

\section{Funding}

This work was supported by the Natural Science Foundation of China (31730090 and 31925037), Hubei Provincial Natural Science Foundation of China (2018CFA020).

\section{Availability of data and materials}

All data generated or analyzed during this study can be made available by the corresponding author upon reasonable request.

\section{Declarations}

\section{Ethics approval}

The animal model and experimental procedures used in this experiment were approved by the Scientific Ethics Committee of Huazhong Agricultural University (approval numbers HZAUSW-2018-015).

Consent for publication

Not applicable.

\section{Competing interests}

The authors declare that they have no competing interests.

\section{Author details}

${ }^{1}$ State Key Laboratory of Agricultural Microbiology, College of Animal Sciences and Technology, Huazhong Agricultural University, Wuhan 430070, Hubei, China. ${ }^{2}$ The Cooperative Innovation Center for Sustainable Pig Production, Wuhan 430070, Hubei, China. ${ }^{3}$ Hubei Provincial Engineering Laboratory for Pig Precision Feeding and Feed Safety Technology, Wuhan 430070, Hubei, China.

Received: 29 October 2020 Accepted: 1 March 2021

Published online: 05 May 2021

\section{References}

1. Spencer TE. Biological roles of uterine glands in pregnancy. Semin Reprod Med. 2014;32(5):346-57.

2. Filant J, Spencer TE. Uterine glands: biological roles in conceptus implantation, uterine receptivity and decidualization. Int J Dev Biol. 2014; 58(2-4):107-16.

3. Gray CA, Bartol FF, Tarleton BJ, Wiley AA, Johnson GA, Bazer FW, Spencer TE. Developmental biology of uterine glands. Biol Reprod. 2001;65(5):1311-23.

4. Bartol FF, Wiley AA, Spencer TE, Vallet JL, Christenson RK. Early uterine development in pigs. J Reprod Fertil. 1993;48(Suppl):99-116.

5. Wu MC, Dziuk PJ. Ovarian influence on uterine growth in prepubertal gilts. $J$ Anim Sci. 1988;66(11):2893-8.

6. Tarleton BJ, Wiley AA, Bartol FF. Neonatal estradiol exposure alters uterine morphology and endometrial transcriptional activity in prepubertal gilts. Domest Anim Endocrinol. 2001;21(2):111-25. 
7. Cooke PS, Spencer TE, Bartol FF, Hayashi K. Uterine glands: development, function and experimental model systems. Mol Hum Reprod. 2013;19(9): 547-58

8. Xu X, Faillace LS, Harding RT, Foxcroft GR, Hunter MG. Evidence that Meishan and large-white hybrid preovulatory follicles may differentially affect oocyte in vitro maturation and fertilization. Anim Reprod Sci. 1998; 51(4):307-19.

9. Christenson RK, Bartol FF, Vallet JL, Wiley AA, Spencer TE. Comparative study of uterine morphogenesis and protein secretion in neonatal white crossbred and Meishan gilts. Biol Reprod. 1997;56(5):1112-9.

10. Jiang X, Lu N, Xue Y, Liu S, Lei H, Tu W, Lu Y, Xia D. Crude fiber modulates the fecal microbiome and steroid hormones in pregnant Meishan sows. Gen Comp Endocrinol. 2019;277:141-7.

11. Chen KL, Madak-Erdogan Z. Estrogen and microbiota crosstalk: should we pay attention? Trends Endocrinol Metab. 2016;27(11):752-5.

12. Nuriel-Ohayon M, Neuman H, Ziv O, Belogolovski A, Barsheshet $Y$, Bloch N et al. Progesterone increases Bifidobacterium relative abundance during late pregnancy. Cell Rep. 2019;27(3):730-6.

13. Cooke PS, Ekman GC, Kaur J, Davila J, Bagchi IC, Clark SG, et al. Brief exposure to progesterone during a critical neonatal window prevents uterine gland formation in mice. Biol Reprod. 2012;86(3):63.

14. Shapira M. Gut microbiotas and host evolution: scaling up Symbiosis. Trends Ecol Evol. 2016;31(7):539-49.

15. Khoruts A, Sadowsky MJ. Understanding the mechanisms of faecal microbiota transplantation. Nat Rev Gastroenterol Hepatol. 2016;13(9):508-16.

16. McCormack UM, Curiao T, Wilkinson T, Metzler-Zebeli BU, Reyer H, Ryan T, et al. Fecal Microbiota Transplantation in Gestating Sows and Neonatal Offspring Alters Lifetime Intestinal Microbiota and Growth in Offspring. mSystems. 2018;3(3):e00134-17.

17. Zhang P, Feng Y, Li L, Ge W, Yu S, Hao Y, et al. Improvement in sperm quality and spermatogenesis following faecal microbiota transplantation from alginate oligosaccharide dosed mice. Gut. 2020;70(1):222-5.

18. Kwolek-Mirek M, Zadrag-Tecza R. Comparison of methods used for assessing the viability and vitality of yeast cells. FEMS Yeast Res. 2014;14(7): 1068-79.

19. Hamilton MJ, Weingarden AR, Sadowsky MJ, Khoruts A. Standardized frozen preparation for transplantation of fecal microbiota for recurrent Clostridium difficile infection. Am J Gastroenterol. 2012;107(5):761-7.

20. Vallet JL, Calderón-Díaz JA, Stalder K, Phillips C, Cushman RA, Miles JR, et al. Litter-of-origin trait effects on gilt development. J Anim Sci. 2016;94(1):96-105.

21. Costea PI, Zeller G, Sunagawa S, Pelletier E, Alberti A, Levenez F, et al. Towards standards for human fecal sample processing in metagenomic studies. Nat Biotechnol. 2017;35(11):1069-76.

22. Fadrosh DW, Ma B, Gajer P, Sengamalay N, Ott S, Brotman RM, et al. An improved dual-indexing approach for multiplexed 16S rRNA gene sequencing on the Illumina MiSeq platform. Microbiome. 2014;2(1):6.

23. Bolyen E, Rideout JR, Dillon MR, Bokulich NA, Abnet CC, Al-Ghalith GA, et al. Reproducible, interactive, scalable and extensible microbiome data science using QIIME 2. Nat Biotechnol. 2019;37(8):852-7.

24. Magoč T, Salzberg SL. FLASH: fast length adjustment of short reads to improve genome assemblies. Bioinformatics. 2011;27(21):2957-63.

25. Edgar RC. UPARSE: highly accurate OTU sequences from microbial amplicon reads. Nat Methods. 2013:10(10):996-8.

26. DeSantis TZ, Hugenholtz P, Larsen N, Rojas M, Brodie EL, Keller K, et al. Greengenes, a chimera-checked $16 \mathrm{~S}$ rRNA gene database and workbench compatible with ARB. Appl Environ Microbiol. 2006;72(7):5069-72.

27. Schloss PD, Westcott SL, Ryabin T, Hall JR, Hartmann M, Hollister EB, et al. Introducing mothur: open-source, platform-independent, communitysupported software for describing and comparing microbial communities. Appl Environ Microbiol. 2009;75(23):7537-41.

28. Douglas GM, Maffei VJ, Zaneveld JR, Yurgel SN, Brown JR, Taylor CM, et al. PICRUSt2 for prediction of metagenome functions. Nat Biotechnol. 2020; 38(6):685-8

29. Franklin MA, Mathew AG, Vickers JR, Clift RA. Characterization of microbial populations and volatile fatty acid concentrations in the jejunum, ileum, and cecum of pigs weaned at 17 vs 24 days of age. J Anim Sci. 2002:80(11):2904-10.

30. Fellows R, Denizot J, Stellato C, Cuomo A, Jain P, Stoyanova E, et al. Microbiota derived short chain fatty acids promote histone crotonylation in the colon through histone deacetylases. Nat Commun. 2018;9(1):105.

31. Dunn WB, Broadhurst D, Begley P, Zelena E, Francis-McIntyre S, Anderson N, et al. Procedures for large-scale metabolic profiling of serum and plasma using gas chromatography and liquid chromatography coupled to mass spectrometry. Nat Protoc. 2011;6(7):1060-83.

32. Simmen RC, Simmen FA, Hofig A, Farmer SJ, Bazer FW. Hormonal regulation of insulin-like growth factor gene expression in pig uterus. Endocrinology. 1990;127(5):2166-74

33. Clarke G, Stilling RM, Kennedy PJ, Stanton C, Cryan JF, Dinan TG. Minireview: gut microbiota: the neglected endocrine organ. Mol Endocrinol. 2014;28(8): 1221-38.

34. Flores $R$, Shi J, Fuhrman $B, X u X$, Veenstra TD, Gail MH, et al. Fecal microbial determinants of fecal and systemic estrogens and estrogen metabolites: a cross-sectional study. J Transl Med. 2012;10:253.

35. Goodrich JK, Waters JL, Poole AC, Sutter JL, Koren O, Blekhman R, et al. Human genetics shape the gut microbiome. Cell. 2014;159(4):789-99.

36. Yuan M, Li D, Zhang Z, Sun H, An M, Wang G. Endometriosis induces gut microbiota alterations in mice. Hum Reprod. 2018:33(4):607-16.

37. Yang L, Bian G, Su Y, Zhu W. Comparison of faecal microbial community of lantang, bama, erhualian, Meishan, xiaomeishan, duroc, landrace, and Yorkshire sows. Asian-Australas J Anim Sci. 2014;27(6):898-906.

38. Uryu H, Tsukahara T, Ishikawa H, Oi M, Otake S, Yamane I, et al. Comparison of Productivity and Fecal Microbiotas of Sows in Commercial Farms. Microorganisms. 2020;8(10):1469.

39. Li SS, Zhu A, Benes V, Costea Pl, Hercog R, Hildebrand F, et al. Durable coexistence of donor and recipient strains after fecal microbiota transplantation. Science. 2016;352(6285):586-9.

40. Lee STM, Kahn SA, Delmont TO, Shaiber A, Esen ÖC, Hubert NA, et al. Antonopoulos DA, Rubin DT, Eren AM: tracking microbial colonization in fecal microbiota transplantation experiments via genome-resolved metagenomics. Microbiome. 2017:5(1):50.

41. Kelleher AM, Peng W, Pru JK, Pru CA, DeMayo FJ, Spencer TE. Forkhead box a2 (FOXA2) is essential for uterine function and fertility. Proc Natl Acad Sci U S A. 2017;114(6):E1018-26.

42. Reardon SN, King ML, MacLean JA, Mann JL, DeMayo FJ, Lydon JP, et al. $\mathrm{CDH} 1$ is essential for endometrial differentiation, gland development, and adult function in the mouse uterus. Biol Reprod. 2012;86(5):141-10.

43. Kiewisz J, Kaczmarek MM, Andronowska A, Blitek A, Ziecik AJ. Gene expression of WNTs, $\beta$-catenin and E-cadherin during the periimplantation period of pregnancy in pigs--involvement of steroid hormones. Theriogenology. 2011;76(4):687-99.

44. Jeong JW, Kwak I, Lee KY, Kim TH, Large MJ, Stewart CL, et al. Foxa2 is essential for mouse endometrial gland development and fertility. Biol Reprod. 2010;83(3):396-403.

45. Kwa M, Plottel CS, Blaser MJ, Adams S. The Intestinal Microbiome and Estrogen Receptor-Positive Female Breast Cancer. J Natl Cancer Inst. 2016;108(8):djw029.

46. Zhuo $Y$, Feng $B$, Xuan $Y, C$ Che $L$, Fang $Z$, Lin $Y$, et al. Inclusion of purified dietary fiber during gestation improved the reproductive performance of sows. J Anim Sci Biotechnol. 2020;11(1):47.

47. van de Wouw M, Boehme M, Lyte JM, Wiley N, Strain C, O'Sullivan O, et al. Short-chain fatty acids: microbial metabolites that alleviate stress-induced brain-gut axis alterations. J Physiol. 2018;596(20):4923-44.

48. Kimura-Todani T, Hata T, Miyata N, Takakura S, Yoshihara K, Zhang XT, et al. Dietary delivery of acetate to the colon using acylated starches as a carrier exerts anxiolytic effects in mice. Physiol Behav. 2020;223:8.

49. den Besten G, van Eunen K, Groen AK, Venema K, Reijngoud D-J, Bakker BM. The role of short-chain fatty acids in the interplay between diet, gut microbiota, and host energy metabolism. J Lipid Res. 2013;54(9):2325-40.

50. Daigle C. Parallels between Postpartum Disorders in Humans and Preweaning Piglet Mortality in Sows. Animals (Basel). 2018:8(2):22.

51. Damm Bl, Forkman B, Pedersen LJ. Lying down and rolling behaviour in sows in relation to piglet crushing. Appl Anim Behav Sci. 2005;90(1):3-20.

52. Koketsu Y, Tani S, lida R. Factors for improving reproductive performance of sows and herd productivity in commercial breeding herds. Porcine Health Manag. 2017;3(1):1. 